\title{
The role of iris pigmentation in the perception of an afterimage
}

\author{
BENJAMIN WALLACE \\ Cleveland State University, Cleveland, Ohio 44115
}

\begin{abstract}
Duration, direction-change frequency, and color-change frequency of an induced afterimage were investigated as a function of iris pigmentation (blue or brown) and the wavelength of a light pulse used to bleach retinal photoreceptors. For blue-irised subjects, a yellow (Wratten 9) pulse produced the longest enduring afterimage while a blue-green (Wratten 44A) pulse produced the shortest enduring afterimage. The reverse was true for brown-irised subjects. Neither of the other dependent measures was affected by iris pigmentation or by pulse color. The afterimage duration results were explained in terms of the possible role of the iris in selectively and differentially filtering various wavelengths of light as a function of its pigmentation.
\end{abstract}

The human iris is a sphincter muscle which opens and closes as a function of the light intensity of a stimulus projected to the eye. At low levels it dilates; at high levels it contracts. As such, the iris controls the amount of light which eventually is projected to the retina through the iris opening, the pupil. This procedure appears to operate in a similar fashion regardless of iris pigmentation, the genetically determined color of the eye (iris).

Recently, however, Coren and Porac (1978) reasoned that since a function of the iris is to prevent light from entering the eye except through the pupil, and since light- or blue-irises do not absorb incoming light as efficiently as brown irises (Walls, 1967), blue irises may have to contend with more stray light within the optic globe than brown irises (Polyak, 1957). As a result, iris pigmentation may have an effect upon certain perceptual phenomena. In fact, Coren and Porac reported that iris pigmentation did affect the perceived magnitude of the Müller-Lyer illusion. Blue-irised individuals reported perceiving a stronger illusion than brown-irised subjects. This outcome was explained as a result of light, or blueirises permitting more scattered light within the eye, which consequently degraded the retinal image of the stimulus object(s). Such degrading is generally theorized to affect Müller-Lyer illusory magnitude (Chiang, 1968).

If iris pigmentation does, indeed, play a significant role in perception, blue-irised individuals and brownirised individuals might respond differently to different wavelengths of light. Specifically, since the iris controls the amount of light which is projected to the

The assistance of Mary Gilbert in this reseach is gratefully acknowledged. Reprint requests should be sent to Benjamin Wallace, Department of Psychology, Cleveland State University, Cleveland, Ohio 44115. retina, it is also possible that selective filtering of light might occur at the iris as a function of light wavelength. As a result, blue-irised individuals may respond differently than brown-irised subjects to some projected colors.

One procedure by which this might be tested is to consider the perception of an afterimage as a function of iris pigmentation and light pulse wavelength. Thus, if a blue or brown iris is exposed to a light pulse, an afterimage will result. However, if the wavelength of the light pulse is varied, it is possible that afterimage perception(s) may vary. In other words, differential filtering of various wavelengths of light might occur at the iris before the light is projected to the retina. The result of such might be the production of a longer duration afterimage for blue-irised subjects compared to brown-irised subjects, especially perhaps if the wavelength of the light is related to the color of the iris. If such is the case, blue-irised individuals would, for example, respond differently than would brown-irised subjects to a blue light source. The role of the present investigation was to determine if iris pigmentation and various wavelengths of light would influence afterimage percepts.

\section{METHOD}

\section{Subjects}

Sixty undergraduates from introductory psychology sections served as subjects. All individuals had normal, uncorrected visual acuity and normal color vision. Half of the subjects were selected for possessing blue-irised eyes; the remainder were selected for having brown irises. Hazel, gray, and black-irised subjects were not employed. The subjects were not aware that iris color was a factor under consideration in the present investigation.

\section{Apparatus}

The experiment was conducted in a $3.1 \times 6.4 \mathrm{~m}$ completely lightproofed room. The stimulus to be observed was produced by a pulse (approximately $1 / 1000 \mathrm{sec}$ in duration) from an elec- 
tronic 2,000 beam $\mathrm{cp} / \mathrm{sec}$ Vivitar (Model 273) flash unit. This unit was mounted on an adjustable stand which could be raised or lowered to permit the light source to be projected to the subjects' eyes from an eye-level position. The actual stimulus size was 6 min visual angle. This was accomplished by totally masking the flash unit so that it would emit light only through the small aperture. In addition, the stimulus was flashed through either a yellow Wratten 9 filter (producing a bluish negative afterimage), a blue-green Wratten 44A filter (producing a yellowish negative afterimage), or a red Wratten 92 filter (producing a greenish negative afterimage). ${ }^{1}$ These particular filters were chosen from a sample of 12 filters (4 of each color) because, when a light pulse was projected through each chosen filter, the stimuli were judged to be equally bright (they were not physically equal in terms of percent of luminous transmission - see Footnote 1). This psychophysical assessment was conducted prior to the experiment with the assistance of three blue-irised and three brown-irised judges. These individuals did not subsequently participate in the experiment. Stability of the subject's head during the experiment was maintained with a Marietta Instruments combination head- and chinrest.

\section{Procedure}

The subjects were led individually, blindfolded, to the experimental room and seated in a chair with a back support and an arm support. Upon the arm support was mounted the headand chinrest. The experimenter then adjusted the stand upon which was mounted the Vivitar unit so that the aperture of the electronic flash was at eye level with the subject. After this adjustment, the room lights were turned off, the blindfold was removed from the subject's eyes, and he/she was permitted to dark adapt for a total of $25 \mathrm{~min}$. Following this period of time, the subject was instructed to gaze in the straight-ahead direction in space to observe a spot of light. The subjects localized this position by fixating toward the voice of the experimenter. A visual fixation point was purposely avoided in order to eliminate any possible interfering influence of such on the eventual afterimage formation of the target pulse. The experimenter then triggered the flash unit to produce the light pulse from a viewing distance of $2.5 \mathrm{~m}$. The flash was red, yellow, or blue-green in color. An equal number of blue- and brown-irised individuals participated in each condition ( 10 per each wavelength of light).

Before the flash was triggered, subjects were instructed to try as hard as possible not to blink. Upon perceiving the flash, the subjects were instructed to report when an afterimage was first visible, any perceived movement of the image, any noticeable color changes of the image, and when the image was no longer visible. Thus, the subjects were instructed to give a continuous oral description of the nature of the produced afterimage. Such descriptions were tape-recorded, and subsequent duration assessments of the afterimage were determined with a stopwatch. Each subject was exposed to a total of three flashes, with a 25 -min intertrial interval to permit the subject to re-dark adapt. A trial was defined as the period of time from the initial observation of an afterimage to the time the initial afterimage was no longer visible. Only first afterimages were assessed in the present investigation. Any subject failing to report perceiving such a first afterimage on any trial was eliminated from data analyses and was substituted with another subject. Six subjects were so replaced, two in each of the three color conditions.

\section{RESULTS}

Three dependent measures were assessed in the present investigation: afterimage duration (in seconds), reported direction change frequency of the afterimage, and reported color change frequency of the afterimage. A preliminary analysis on each of these measures indicated the absence of a significant trials effect. As a consequence, subsequent analyses of the
Table 1

Mean Duration (in Seconds) of an Afterimage as a Function of Iris Pigmentation and Filter Color

\begin{tabular}{|c|c|c|c|c|c|c|}
\hline \multirow{3}{*}{$\begin{array}{l}\text { Iris } \\
\text { Pigmen- } \\
\text { tation }\end{array}$} & \multicolumn{6}{|c|}{ Filter Color } \\
\hline & \multicolumn{2}{|c|}{ Red } & \multicolumn{2}{|c|}{ Yellow } & \multicolumn{2}{|c|}{ Blue-Green } \\
\hline & Mean & SD & Mean & SD & Mean & SD \\
\hline Blue & 32.33 & 7.36 & 43.71 & 6.78 & 13.89 & 6.95 \\
\hline Brown & 34.33 & 8.74 & 20.42 & 6.42 & 42.88 & 9.16 \\
\hline
\end{tabular}

Note-Filters used were Wratten 92 (red), 9 (yellow), and $44 \mathrm{~A}$ (blue-green).

measures were performed for data averaged over the three exposure trials. An analysis of variance was performed on these dependent measures as a function of iris pigmentation (blue or brown) and filter color (red, yellow, or blue-green).

For the first dependent measure, afterimage duration, iris pigmentation significantly interacted with filter color $[F(2,54)=4.04, p<.02]$. The mean duration values for the various conditions are depicted in Table 1. For the subjects with blue irises, the afterimage produced by the yellow filter endured for a significantly longer period of time (NewmanKeuls, $p<.05$ ) than when produced by either the red filter or the blue-green filter. Also, afterimage duration was significantly shorter in duration (Newman-Keuls, $p<.01$ ) when produced by the bluegreen filter than when produced by the red filter.

When subjects possessed brown irises, an afterimage endured significantly longer (Newman-Keuls, $\mathrm{p}<.01$ ) when it was produced by the blue-green filter compared to when it was produced by either the red or yellow filter. Afterimage duration was also significantly shorter in duration (Newman-Keuls, $p<.01$ ) when produced by the yellow filter than when it was produced by the red filter. In addition, significant differences (Newman-Keuls, $p<.05$ ) were found to exist between blue- and brown-irised subjects for afterimage duration when the filter was either yellow or blue-green. When the filter color was red, afterimage duration for blue- and brown-irised subjects was not significantly different.

With regard to reported direction change frequency as a function of iris pigmentation and filter color, neither factor affected this dependent measure. The mean direction and color change frequency values for the various experimental conditions are depicted in Tables 2 and 3, respectively. In the present investigation, a color change was defined as perceiving a color other than the filter color. Thus, for example, when the filter was red, the typical afterimage perceived and reported was greenish in nature. This constituted one color change. However, even when the negative afterimage was initially perceived as greenish, it often would be reported to change to other colors, such as yellow or white. In fact, many subjects did report some color changes. However, when the filter was red, all subjects, regardless of 
Table 2

Mean Direction Change Frequency of an Afterimage as a Function of Iris Pigmentation and Filter Color

\begin{tabular}{|c|c|c|c|c|c|c|}
\hline \multirow{3}{*}{$\begin{array}{c}\text { Iris } \\
\text { Pigmen- } \\
\text { tation }\end{array}$} & \multicolumn{6}{|c|}{ Filter Color } \\
\hline & \multicolumn{2}{|c|}{ Red } & \multicolumn{2}{|c|}{ Yellow } & \multicolumn{2}{|c|}{ Blue-Green } \\
\hline & Mean & SD & Mean & SD & Mean & $\mathrm{SD}$ \\
\hline Blue & 1.70 & .58 & 1.70 & .71 & 1.83 & .81 \\
\hline Brown & 1.03 & .74 & 1.47 & .76 & 1.40 & .87 \\
\hline
\end{tabular}

Note-Filters used were Wratten 92 (red), 9 (yellow), and 44A (blue-green).

Table 3

Mean Color Change Frequency of an Afterimage as a Function of Iris Pigmentation and Filter Color

\begin{tabular}{|c|c|c|c|c|c|c|}
\hline \multirow{3}{*}{$\begin{array}{c}\text { Iris } \\
\text { Pigmen- } \\
\text { tation }\end{array}$} & \multicolumn{6}{|c|}{ Filter Color } \\
\hline & \multicolumn{2}{|c|}{ Red } & \multicolumn{2}{|c|}{ Yellow } & \multicolumn{2}{|c|}{ Blue-Green } \\
\hline & Mean & SD & Mean & $\mathrm{SD}$ & Mean & SD \\
\hline Blue & 1.27 & .55 & 2.13 & .76 & 1.83 & 1.06 \\
\hline Brown & 1.47 & .63 & 1.57 & .53 & 1.50 & .50 \\
\hline
\end{tabular}

Note-Filters used were Wratten 92 (red), 9 (yellow), and 44A (blue-green).

iris color, initially reported the negative afterimage to be greenish in appearance. When the filter was yellow, the negative afterimage was reported to be bluish. For the blue-green filter, the negative afterimage was reported to be yellowish. These, of course, are the expected afterimage colors for the wavelengths of light employed in the present study (Hurvich \& Jameson, 1957; Jameson \& Hurvich, 1955; Uttal, 1973).

\section{DISCUSSION}

As the data of the present investigation indicate, iris pigmentation appears to have had an effect on the reported duration of an induced afterimage. Specifically, for a blue-irised eye, a light pulse projected through a yellow filter produced the longest persisting afterimage while a light pulse projected through a blue-green filter produced the shortest duration afterimage. For a brown-irised eye, a light pulse projected through a blue-green filter produced the longest persisting afterimage while a light projected through a yellow filter produced the shortest duration afterimage.

While the sensory physiology that underlies the aforementioned phenomenon is unclear, it does appear that iris color influences afterimage duration for specific wavelengths of light. One explanation for this may relate to the ability of the iris to selectively filter specific wavelengths of light as they are projected to the retina. As a result, although the major function of the iris is to regulate the intensity or amount of light projected to the retina, a secondary function appears to be in selectively filtering that light. Thus, for a blue-pigmented iris, yellow light appears to be less filtered than either a red or a bluegreen light. The result of such is to produce a longer persisting afterimage. A similar process would result for brown-irised individuals perceiving a blue-green light.

The shortest duration afterimage also appears to be related to iris pigmentation. Specifically, when a light pulse is blue-green, this produces the shortest duration afterimage for a blue iris; a yellow light produces the shortest duration afterimage for a brown iris. Thus, it appears that when a light pulse approximates in wavelength the color of the iris, the greatest amount of light and/or wavelength filtering occurs before the light arrives to the retina. Such filtering may, in turn, produce a degraded and less enduring afterimage.

It is interesting to note that although the various light pulses were judged to be psychophysically equal in brightness when the luminous transmission levels of each employed pulse color were quite different (see Footnote 1), this appeared to have no effect upon afterimage reports. Only iris pigmentation appeared to have an effect on such reports and only for reports of afterimage duration. Whether the iris is, in fact, a selective filter for various wavelengths of light projected to the retina and that this filtering is related to iris pigmentation is unclear. However, the present study presents tentative evidence to indicate that this may be the case.

\section{REFERENCES}

Chiang, C. A new theory to explain geometrical illusions produced by crossing lines. Perception \& Psychophysics, 1968, 3, 174-176.

Coren, S., \& Porac, C. Iris pigmentation and visual-geometric illusions. Perception, 1978, 7, 473-477.

Hurvich, L. M., \& Jameson, D. An opponent-process theory of color vision. Psychological Review, 1957, 64, 384-404.

JAMESON, D., \& HURvich, L. M. Some quantitative aspects of an opponent-colors theory. I. Chromatic responses and spectral saturation. Journal of the Optical Society of America, 1955, 45, 546-552.

OSA CommitTEE on Colorimetry. The science of color. New York: Crowell, 1953.

Polyak, S. The vertebrate visual system. Chicago: University of Chicago Press, 1957.

UTTAL, W. R. The psychobiology of sensory coding. New York: Harper and Row, 1973.

WALLS, G. L. The vertebrate eye and its adaptive radiation. New York: Hafner, 1967.

\section{NOTE}

1. The Wratten 9 filter has a dominant wavelength of $582.8 \mathrm{~nm}$, an excitation purity of $90.5 \%$, and a $82.4 \%$ luminous transmission. The Wratten $44 \mathrm{~A}$ filter has a dominant wavelength of $491.9 \mathrm{~nm}$, an excitation purity of $76.0 \%$, and $10.1 \%$ luminous transmission. The Wratten 92 filter has a dominant wavelength of $646.2 \mathrm{~nm}$, an excitation purity of $100.0 \%$, and a $4.8 \%$ luminous transmission. These colorimetric specifications are based on the $1931 \mathrm{CIE}$ standard colorimetric and luminosity data (OSA Committee on Colorimetry, 1953).

(Received for publication July 31, 1979; revision accepted October 1, 1979.) 